\title{
Wakefields due to surface waves in a beam pipe with a periodic rough surface
}

\author{
A. Mostacci* and F. Ruggiero \\ CERN, Geneva, Switzerland
}

\begin{abstract}
M. Angelici, M. Migliorati, L. Palumbo, and S. Ugoli ${ }^{\dagger}$
Dipartimento di Energetica-Universitá La Sapienza, Roma, Italy

(Received 14 July 2000; revised manuscript received 22 February 2002; published 12 April 2002)

The problem of the wakefields generated by an ultrarelativistic particle traveling in a long beam tube with a periodic rough surface has been revisited by means of a standard theory based on the hybrid modes excited in a periodically corrugated rectangular waveguide. Slow surface waves synchronous with the particle can be excited in the structure, producing wakefields whose frequency and amplitude depend on the depth of the corrugation. We apply our results to the case of the CERN Large Hadron Collider beam screen and the Linac Coherent Light Source undulator.
\end{abstract}

DOI: 10.1103/PhysRevSTAB.5.044401

PACS numbers: 41.75.-i, 41.20.-q

\section{INTRODUCTION}

The effect of surface roughness is a relatively new subject, arising in the design of machines with extremely short bunches of the order of tens of microns. In this case, in fact, the surface roughness may be a source of wakefields which might significantly increase the beam emittance and the energy spread. Recently, a corrugation of the Large Hadron Collider (LHC) beam pipe has been proposed at CERN in order to reduce the reflectivity of the walls and therefore decrease the heat load on the cold dipole beam screen due to photoelectrons accelerated by the proton beam [1].

The low frequency coupling impedance due to the wall surface roughness has been estimated in Ref. [2]. A different approach, using a small angle approximation in the wall profile, has been developed and gives estimations of the coupling impedance based on the statistical properties of the surface (see, for example, [3]). Small corrugations in the beam pipe were already treated as perturbations of the wall profile in [4], and estimates were given for the electromagnetic field and the total energy loss up to the second order in the perturbation parameter; Ref. [5] reformulates such an expansion with special focus on the resonant frequencies and on a square-wave wall distortion. A dielectric layer model has also been proposed [6]: the roughness is replaced by a thin dielectric layer at the waveguide walls, which supports a surface wave. The synchronous mode resulting from the interaction of a particle beam and a corrugated waveguide has been already studied in the framework of the synchronous wave pickup proposed in [7].

In this paper we review the problem of the wakefields produced by an ultrarelativistic charge traveling inside a beam tube with a periodic corrugation, making use of a

\footnotetext{
*Also with Dipartimento di Energetica-Universitá La Sapienza, Roma.

†Now with Agilent Co.
}

standard theory based on the hybrid modes propagating in the waveguide. We first derive the dispersion relation for the fields and study the frequency where the synchronous wave can be excited. Then, through the reciprocity principle we get the amplitude of the fields excited by the charge. The resulting wakefield for a point charge disagrees with previous estimates [6], based on the dielectric layer model. An application of our results to the case of the LHC beam screen and of the Linac Coherent Light Source (LCLS) undulator [8] is finally discussed.

\section{THE METHOD}

Let us consider the periodically corrugated rectangular waveguide sketched in Figs. 1 and 2, with $a$ being the dimension of the corrugated side ( $x$ axis) and $b$ the distance (along the $y$ axis) between the corrugations on the opposite faces. We model the wall roughness as a series of periodic (with period $L$ ) rectangular obstacles of height $h$ and thickness $t$. The beam travels along the $z$ axis; we assume $t \ll L$ and $L \ll \lambda$, where $\lambda$ is the wavelength of the electromagnetic fields. We neglect Ohmic losses in the material.

Following [9], we consider a rectangular pipe with corrugations at two opposite sides. The periodicity of the geometry along $z$ allows the use of Floquet's theorem which implies a field solution independent of the period $L$ (obtained from a single cell). For the reader's convenience, we first apply the method to the case of a charge traveling on axis (longitudinal problem); the solution for the transverse case (off-axis charge) is given in Appendix E. In order to find the longitudinal wake function per unit length [10], we go through the following steps. We first solve the homogeneous problem, thus finding the modes propagating in the corrugated waveguide (Sec. III), then we apply the reciprocity principle including the sources (Sec. IV). Finally, we calculate the coupling impedance and wake function (Sec. V). Throughout the paper, we use $\left(\xi_{x}, \xi_{y}, \xi_{z}\right)$ for the wave numbers of the field in a rough waveguide, 


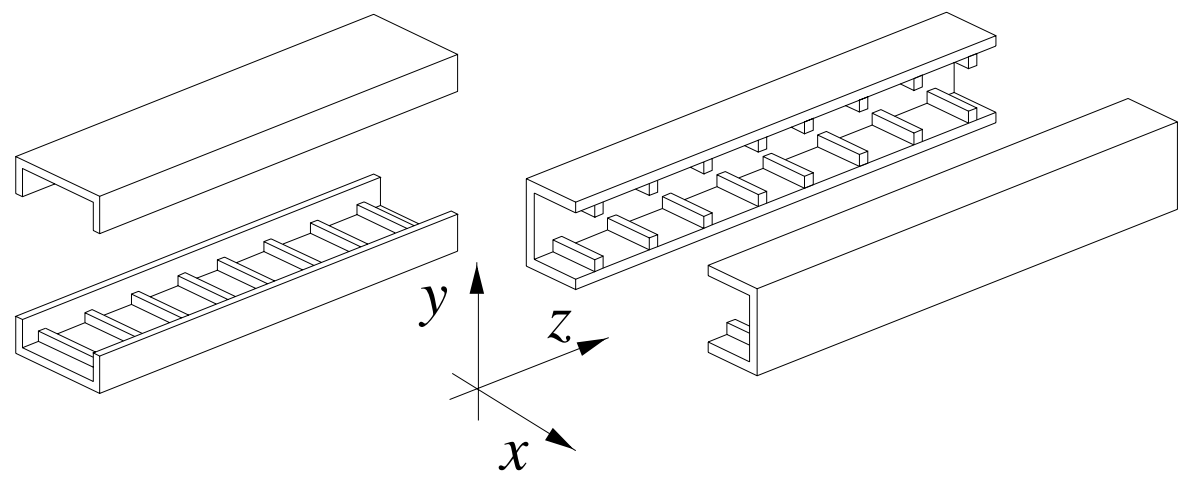

FIG. 1. Relevant geometry.
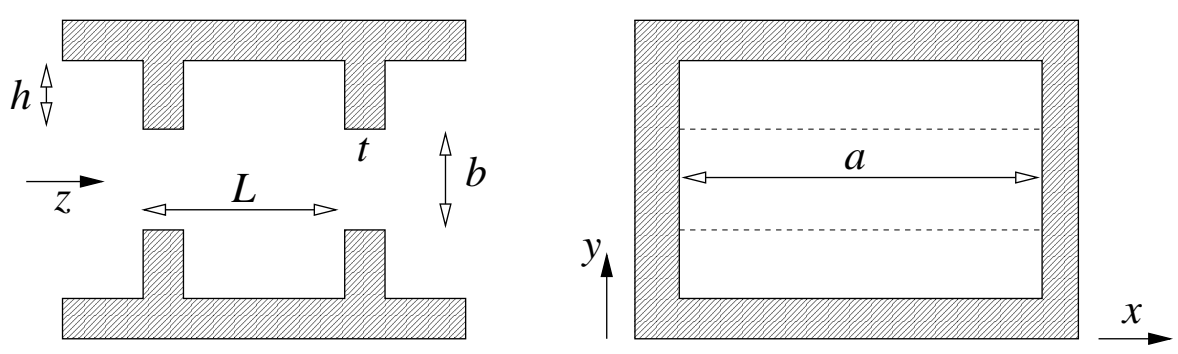

FIG. 2. Schematic view of the waveguide and notations adopted.

$\left(k_{x}, k_{y}, k_{z}\right)$ for a smooth waveguide, and $\left(K_{x}, K_{y}\right)$ for the propagation inside the corrugation that is in the region where $|y|>b / 2$.

\section{HOMOGENEOUS PROBLEM (ON-AXIS CHARGE)}

Since we assumed $L \ll \lambda$, the fields inside the corrugation do not depend on the $z$ variable and, due to the rectangular geometry, they can be written as

$E_{z}^{c}=-j \omega \mu_{0} \sum_{n} B_{n} \sin \left[K_{y n}(b / 2+h-|y|)\right] \cos \left(K_{x n} x\right)$,

$E_{y}^{c}=0$,

$H_{z}^{c}=0$,

$H_{y}^{c}=\frac{\pi}{a} \sum_{n} n B_{n} \sin \left[K_{y n}(b / 2+h-|y|)\right] \sin \left(K_{x n} x\right)$,

$H_{x}^{c}=-K_{y n} \sum_{n} B_{n} \cos \left[K_{y n}(b / 2+h-|y|)\right] \cos \left(K_{x n} x\right)$,

where $B_{n}$ is the field amplitude (depending on the sources),

$$
\begin{gathered}
K_{y n}=\sqrt{(\omega / c)^{2}-K_{x n}^{2}} \text { and } K_{x n}=k_{x n}=n \pi / a, \\
\text { with } n=1,3,5, \ldots
\end{gathered}
$$

Such fields satisfy the boundary condition $E_{z}^{c}=0$ at $x=$ $\pm a / 2$ and $y= \pm(b / 2+h)$. The fields of interest in the internal region of the waveguide can be derived from the magnetic Hertz potential $\boldsymbol{\Pi}=\hat{\boldsymbol{x}} \Pi_{x}$ alone:

$$
\begin{aligned}
\boldsymbol{E} & =-j \omega \mu_{0} \nabla \boldsymbol{\Pi}, \\
\boldsymbol{H} & =\left(k^{2}+\nabla \nabla \cdot\right) \boldsymbol{\Pi} .
\end{aligned}
$$

As shown in Appendix A, depending on the symmetry there are four possible expressions for $\Pi_{x}$, namely, $\Pi_{x i}$ with $i=1, \ldots, 4$. In our case, with the charge traveling along the $z$ axis, only $\Pi_{x i}$ corresponding to $i=2$ has to be considered; it gives a nonvanishing $E_{z}$ on the beam axis. A $y$-directed magnetic Hertz potential is excluded in our analysis since it would produce an $E_{x}$ vanishing on the corrugation, because of continuity over the boundary, as in a smooth conducting rectangular waveguide. Again for continuity reasons $K_{x}$ does not change going from the corrugation to the waveguide: $\xi_{x}=K_{x n}=k_{x n}$. Eventually we can write

$$
k_{x n}^{2}+\xi_{y}^{2}+\xi_{z}^{2}=(\omega / c)^{2} .
$$

The field is continuous over the boundary, i.e.,

$$
E_{z}^{c}=E_{z} \quad \text { and } \quad H_{x}^{c}=H_{x} \quad \text { for } y= \pm b / 2,
$$

and thus (considering only fields derived from $\Pi_{x 2}$ )

$$
K_{y n} \tan \left(K_{y n} h\right)=\xi_{y} \cot \left(\xi_{y} b / 2\right) .
$$

Equation (6) is usually referred to as the dispersion relation; for each value of $K_{y n}$, it has an infinite number of solutions. 
In the limit of a smooth waveguide $(h \rightarrow 0), E_{z}^{c}$ and $H_{x}^{c}$ vanish in Eqs. (5) and we recover the well-known condition $\xi_{y}=k_{y}=m \pi / b$, with $m=1,3,5, \ldots$.

For finite values of $h$, solving numerically Eq. (6) and plugging the resulting value for $\xi_{y}$ in Eq. (4), we get the wave number $\xi_{z}$. A convenient graphical representation for the propagation behavior of these waves is the Brillouin diagram; to show the basic features of the solution, we study the case of a square waveguide of side $a$ and plot the normalized wave number $\xi_{z} a$ as a function of the normalized frequency $a / \lambda$ in Fig. 3 .

The dispersion curve of a smooth waveguide (dashed line) at very high frequencies tends asymptotically to the wave number of an ultrarelativistic beam $\omega / c=2 \pi / \lambda$ (dot-dashed line), but it never crosses it. However, due to the corrugation, there may be a crossing of the dispersion curves at particular frequencies where the waveguide mode and the beam are synchronous (that is, $\xi_{z}=\omega / c$ ), and a coherent exchange of energy is possible. These crossing frequencies depend on the depth of the corrugation $h$. When $\xi_{z}=\omega / c$, from Eq. (4)

$$
\xi_{y}^{2}=-k_{x n}^{2} \Rightarrow \xi_{y}=j k_{x n} .
$$

If $h$ is small with respect to $\lambda$ and to the waveguide dimension $a$, we may also write

$$
K_{y n} \tan \left(K_{y n} h\right) \approx K_{y n}^{2} h .
$$

Then using the definition of $K_{y n}$ [Eq. (2)], we rewrite the dispersion relation Eq. (6) as

$$
\left(\frac{2 \pi \overline{f_{n}}}{c}\right)^{2}-k_{x n}^{2}=\frac{k_{x n}}{h} \operatorname{coth}\left(\frac{b}{2} k_{x n}\right)
$$

thus obtaining the crossing frequencies

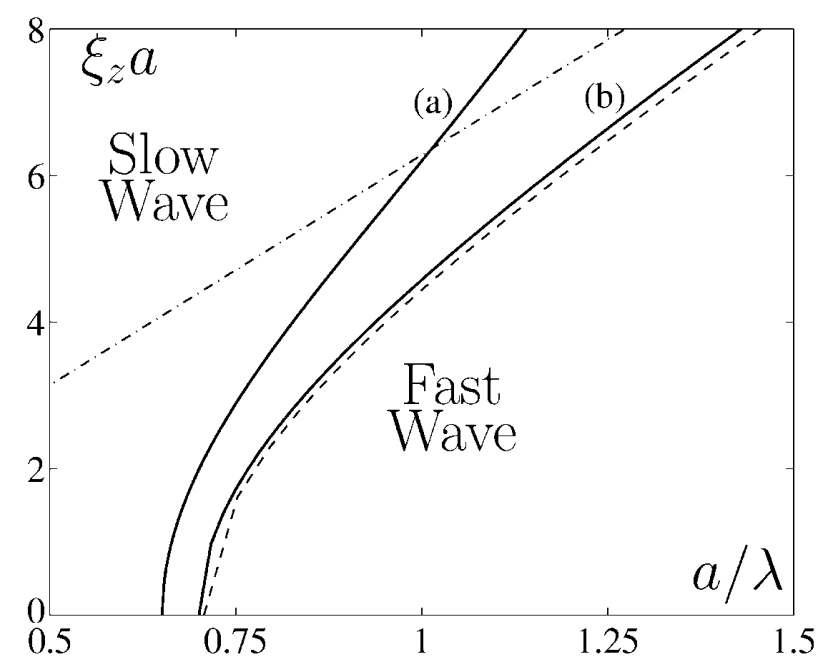

FIG. 3. Dispersion diagram for a square waveguide of side $a$ with corrugation on two opposite sides. The solid lines are obtained from Eq. (6): line (a) is for $h / a=0.1$, while line (b) is for $h / a=0.01$. The dashed line corresponds to the unperturbed $\mathrm{TM}_{11}$ mode of the smooth waveguide and the dot-dashed line represents an ultrarelativistic beam.

$$
\overline{f_{n}}=\frac{c}{2 \pi} \sqrt{k_{x n}^{2}+\frac{k_{x n}}{h} \operatorname{coth}\left(k_{x n} \frac{b}{2}\right)} .
$$

For very small $h$, the second term in the square root dominates, resulting in a behavior $\overline{f_{n}} \propto 1 / \sqrt{h}$, analogous to the case of a pipe covered with a dielectric layer of thickness $h$ [6].

The modes propagating in the corrugated waveguide are called hybrid modes [9], since they can be derived by a superposition of the standard TE and TM modes (along the $z$ axis). The hybrid modes in general do not satisfy an orthogonality condition; physically this means that they are coupled to each other. Since the coupling coefficients are proportional to the height of the corrugation $h$, for very small corrugation depths the modes are practically decoupled.

The minimum frequency beyond which a mode can propagate in the guide (namely, its cutoff frequency $f_{c}$ ) depends on the corrugation depth as well. Mathematically the cutoff frequency is found by imposing the condition $\xi_{z}=0$, which implies $\xi_{y}=\sqrt{(\omega / c)^{2}-k_{x n}^{2}}=K_{y n}$ [from Eq. (4)]. The dispersion relation becomes

$$
\begin{gathered}
K_{y n} \tan \left(K_{y n} h\right)=K_{y n} \cot \left(K_{y n} b / 2\right), \\
\text { that is, } \cos \left(K_{y n} \frac{2 h+b}{2}\right)=0 .
\end{gathered}
$$

Solving for $K_{y n}$ and using Eq. (2), it is straightforward to get

$$
\begin{gathered}
f_{c}=\frac{c}{2 \pi} \sqrt{\left(\frac{n \pi}{a}\right)^{2}+\left(\frac{m \pi}{2 h+b}\right)^{2}} \\
\text { with } n=1,3,5, \ldots, \text { and } m=1,3,5, \ldots
\end{gathered}
$$

For frequencies higher than cutoff, the solution of Eq. (6) combined with Eq. (4) gives real values for $\xi_{z}$, while $\xi_{z}$ is purely imaginary for lower frequencies. In the limit of $h \rightarrow 0$, we find the well-known cutoff frequencies for the modes of a smooth waveguide. Equation (12) concerns only the subset of modes obtainable from $\Pi_{x 2}$.

Moreover, for small $h$ we can write a formula for $\xi_{z}$ as a function of the wave number $k_{z}$ of the smooth waveguide (see Appendix B):

$$
\xi_{z} \approx k_{z}+\frac{2 K_{y n}}{k_{z} b} \tan \left(K_{y n} h\right) .
$$

Since $k_{z}$ and $K_{y n}$ are both depending on the frequency $f$, it is nontrivial to get Eq. (10) directly from Eq. (13).

If the wave number of our hybrid mode falls in the region below (above) the dot-dashed line in Fig. 3, the wave is usually referred to as fast (slow) because its phase velocity is larger (smaller) than the speed of light. Moreover, for a rough waveguide $\xi_{z}$ can exceed the wave number of the smooth one, meaning that the solution $\xi_{y}$ of Eq. (6) is purely imaginary [see Eq. (4)]. In that case the wave is said to be a surface wave, because it is exponentially damped in the $y$ direction. 


\section{INCLUDING THE SOURCES}

Having solved the homogeneous problem, i.e., having derived the modes of the structure, the field generated by a point charge can be found by means of the Lorentz reciprocity principle [11]. Referring to Appendix C, we may write

$$
\begin{aligned}
&-\sum_{m} b_{m} e^{-j\left(\xi_{z n}-\xi_{z m}\right) z} A_{n m}- \\
& \sum_{m} a_{m} e^{-j\left(\xi_{z n}+\xi_{z m}\right) z} B_{n m}=\int_{V} \boldsymbol{J} \cdot \boldsymbol{E}_{n}^{+} d V, \\
&-\sum_{m} a_{m} e^{-j\left(\xi_{z n}-\xi_{z m}\right) z} A_{n m}- \\
& \sum_{m} b_{m} e^{-j\left(\xi_{z n}+\xi_{z m}\right) z} B_{n m}=\int_{V} \boldsymbol{J} \cdot \boldsymbol{E}_{n}^{-} d V,
\end{aligned}
$$

where $\boldsymbol{J}$ is the spectral current density of a point charge traveling on axis $\left(\hat{z}_{o}\right.$ unit vector along the $z$ axis),

$$
\boldsymbol{J}(x, y, z ; \omega)=q \delta(x) \delta(y) e^{-j z \omega / \beta c} \hat{z}_{o},
$$

$a_{n}\left(b_{n}\right)$ is the coefficient of the expansion of the field using the forward (backward) propagating modes of the structure $\boldsymbol{E}_{n}^{+}, \boldsymbol{H}_{n}^{+}\left(\boldsymbol{E}_{n}^{-}, \boldsymbol{H}_{n}^{-}\right)$. Denoting by $S_{c}$ the cross section of the guide,

$$
\begin{aligned}
& A_{n m}=\int_{S_{c}}\left(\boldsymbol{e}_{t n} \times \boldsymbol{h}_{t m}+\boldsymbol{e}_{t m} \times \boldsymbol{h}_{t n}\right) \cdot \hat{z}_{o} d S_{c}, \\
& B_{n m}=\int_{S_{c}}\left(\boldsymbol{e}_{t n} \times \boldsymbol{h}_{t m}-\boldsymbol{e}_{t m} \times \boldsymbol{h}_{t n}\right) \cdot \hat{z}_{o} d S_{c},
\end{aligned}
$$

where $\boldsymbol{e}_{t}$ and $\boldsymbol{h}_{t}$ are the transverse modal function, i.e., the transverse behavior of the mode field. For instance in a smooth waveguide, where the modes are orthogonal, it holds

$$
A_{n m}=2 \delta_{n m} \quad \text { and } \quad B_{n m}=0,
$$

resulting in well-known relations for $a_{n}, b_{n}$ [11] $\left(\delta_{n m}\right.$ stands for the Kronecker's symbol).

In a rough waveguide the (hybrid) modes are no longer orthogonal. Nevertheless only the mode synchronous with the beam can exchange energy over an infinite interaction length [10] (surfing effect), thus we may treat modes one by one; moreover the coupling itself goes to zero in the limit $h \rightarrow 0$ that we are presently interested in. In this case Eqs. (14) become

$$
\begin{aligned}
& a_{n} A_{n n}=-\int_{V} \boldsymbol{J} \cdot \boldsymbol{E}_{n}^{-} d V \text { and } \\
& b_{n} A_{n n}=-\int_{V} \boldsymbol{J} \cdot \boldsymbol{E}_{n}^{+} d V .
\end{aligned}
$$

Since, as shown in Appendix D,

$$
A_{n n}=\omega \mu_{0}\left|\xi_{z n}\right|\left(k^{2}-k_{x n}^{2}\right) a\left[\frac{b}{2}-\frac{\sin \left(\xi_{y} b\right)}{2 \xi_{y}}\right],
$$

and

$$
\int_{V} \boldsymbol{J} \cdot \boldsymbol{E}_{n}^{ \pm} d V= \pm j q \omega \mu_{0} \xi_{y} 2 \pi \delta\left(\frac{\omega}{\beta c} \pm \xi_{z n}\right),
$$

the electric field has a resonant behavior around the crossing frequencies:

$$
\begin{aligned}
E_{z}(x, y, z ; \omega)= & \\
& -2 \pi \beta \frac{q Z_{0}\left[\delta\left(\frac{\omega}{\beta c}-\xi_{z n}\right)+\delta\left(\frac{\omega}{\beta c}+\xi_{z n}\right)\right]}{a\left[b / 2-\sin \left(\xi_{y} b\right) / 2 \xi_{y}\right]} \\
& \times \frac{\xi_{y}^{2}}{k^{2}-k_{x n}^{2}} \cos \left(k_{x n} x\right) \cos \left(\xi_{y} y\right) e^{-j z \omega / \beta c},
\end{aligned}
$$

where $\delta$ is the Dirac delta function, $c$ is the light velocity in vacuum, $k=\omega / c, \beta$ is the relativistic factor, $Z_{0}$ is the free-space characteristic impedance, and $\xi_{y}$ is obtained by solving Eq. (6). The field is non-null only at the crossing frequencies $\bar{f}$ given by Eq. (10), i.e., those such that $\xi_{z}=\omega / \beta c$. It is worth noting that all the frequency dependent quantities in Eq. (21), i.e., $\xi_{y}$ and $k$, have now to be computed at the crossing frequency because of the Dirac functions; thus we may simplify Eq. (21) since for $h \ll \lambda$ and from Eq. (6):

$$
\frac{\xi_{y}^{2}}{k^{2}-k_{x n}^{2}}=-\frac{k_{x n} h}{\operatorname{coth}\left(k_{x n} b / 2\right)} \text {. }
$$

The synchronism between the field in the waveguide and the beam is possible because of the slowing effect due to the surface roughness.

For ultrarelativistic particles $(\beta=1)$ and small $h(h \ll$ $\lambda)$, the electric field at the lowest frequency $(n=1)$ is

$$
\begin{aligned}
E_{z}(x, y, z ; \omega) \sim & -4 \pi^{2} q Z_{0} \frac{h}{a} \frac{1}{a b}\left\{\operatorname{coth}\left(\frac{\pi}{2} \frac{b}{a}\right)\left[\frac{\sinh (\pi b / a)}{\pi b / a}-1\right]\right\}^{-1} \\
& \times \cos \left(\frac{\pi}{a} x\right) \cosh \left(\frac{\pi}{a} y\right) e^{-j z \omega / c}\left[\delta\left(\omega / c-\xi_{z 1}\right)+\delta\left(\omega / c+\xi_{z 1}\right)\right],
\end{aligned}
$$

providing that Eq. (7) holds for $\xi_{y}$ at the crossing frequency. First of all, $E_{z}$ has a phase difference of $\pi$ relative to the charge field (the minus sign), meaning that it is a decelerating field. The field is confined in the waveguide region near the corrugated wall (it is exponentially growing for $y \rightarrow \pm b / 2$ ), as we expect for a surface wave. The height of the corrugation fixes not only the resonant frequency through $\xi_{z 1}$, but also the field amplitude through the factor $h / a$ : the field decreases in amplitude and increases in frequency as $h$ decreases. 
A structure of finite length $\ell$ will result in a spread of frequencies that can be excited, since

$$
2 \pi \delta\left(\omega / \beta c \pm \xi_{z n}\right) \rightarrow \ell \frac{\sin \left[\left(\omega / \beta c \pm \xi_{z n}\right) \ell / 2\right]}{\left(\omega / \beta c \pm \xi_{z n}\right) \ell / 2}
$$

\section{LONGITUDINAL COUPLING IMPEDANCE AND WAKE FUNCTION}

Following the standard definition of the (specific) longitudinal coupling impedance [10]

$$
\frac{d Z(\omega)}{d z}=-\frac{1}{q} E_{z}(x=0, y=0, z, \omega) e^{j z \omega / c},
$$

from Eq. (23) we get the (real) coupling impedance due to the surface wave:

$$
\begin{aligned}
\frac{d Z(\omega)}{d z}= & 4 \pi^{2} Z_{0} \frac{h}{a} \frac{1}{a b}\left\{\operatorname{coth}\left(\frac{\pi}{2} \frac{b}{a}\right)\left[\frac{\sinh (\pi b / a)}{\pi b / a}-1\right]\right\}^{-1} \\
& \times\left[\delta\left(\omega / c-\xi_{z 1}\right)+\delta\left(\omega / c+\xi_{z 1}\right)\right] .
\end{aligned}
$$

It is straightforward now to get the wake function for unit length and for a point charge [10], i.e.,

$$
\frac{d w(\tau)}{d z}=\frac{H(\tau)}{\pi} \int_{-\infty}^{\infty} \frac{d Z(\omega)}{d z} e^{j \omega \tau} d \omega
$$

where $\tau$ is the time distance of the trailing charge from the leading one and $H(\tau)$ is the Heaviside function. Eventually we get

$$
\frac{d w(\tau)}{d z}=w_{0}(a, b, h) \cos \left(2 \pi \bar{f}_{1} \tau\right) H(\tau)
$$

where

$$
\begin{aligned}
w_{0}(a, b, h)= & 8 \pi \frac{Z_{0} c}{a b} \frac{h}{a} \\
& \times\left\{\operatorname{coth}\left(\frac{\pi}{2} \frac{b}{a}\right)\left[\frac{\sinh (\pi b / a)}{\pi b / a}-1\right]\right\}^{-1} .
\end{aligned}
$$

To get the wake function for a bunch, one has to perform the convolution of Eq. (28) with the bunch spectrum. For instance, the wake function is

$$
\frac{d W(\tau)}{d z}=w_{0}(a, b, h) \int_{0}^{\infty} \frac{e^{-(t-\tau)^{2} / 2 \sigma^{2}}}{\sqrt{2 \pi} \sigma} \cos \left(2 \pi \bar{f}_{1} t\right) d t
$$

for a Gaussian bunch of rms bunch length $\sigma$, and this integral has to be performed numerically.

\section{A. Application to the LHC beam screen}

The amplitude $w_{0}(a, b, h)$ of the sinusoidal wake function for an LHC-like geometry ${ }^{1}\left(a=3.6 \times 10^{-2} \mathrm{~m}, b=\right.$ $4.3 \times 10^{-2} \mathrm{~m}$, and $\left.h=30 \mu \mathrm{m}\right)$ is $\simeq 0.3 \mathrm{~V} \mathrm{pC}^{-1} \mathrm{~m}^{-1}$

\footnotetext{
${ }^{1}$ In the LHC the corrugations are on the vertical, shorter sides.
}

and the first crossing frequency $\overline{f_{1}}$ is $83 \mathrm{GHz}$ (and $h \ll \lambda$, as assumed). Since the rms bunch length is $\sigma \approx 7.5 \mathrm{~cm}$, the bunch cutoff frequency $c /(2 \pi \sigma)$ is of the order of $1 \mathrm{GHz}$, i.e., nearly a factor of 100 lower than the wake resonant frequency (that is the crossing frequency).

Until now we have shown that the wakefield due to the synchronous mode has a resonant behavior and the quality factor $Q$ of such a resonance is infinite, since we have assumed an infinite interaction length, no Ohmic losses, and an ideal geometry. This is clearly an approximation and, to estimate the threshold of the longitudinal instability, we will consider the standard resonator model [12]. We assume that the amplitude of the wake function given by Eq. (29) is produced by a high (but finite) $Q$ resonator impedance with a shunt resistance $R_{s}$ given by

$$
R_{s}=\frac{Q w_{0} L_{0}}{2 \pi \overline{f_{1}}}=1.6 \times 10^{4} Q \Omega
$$

with a finite $Q$ value. The LHC parameters we will use are given in Table I.

The impedance at frequencies well below the resonator frequency (for example, at the frequency $f_{\text {bunch }}=$ $c / 2 \pi \sigma$ associated with the bunch length) is inductive and such that

$$
|Z / n| \approx \frac{R_{s}}{Q} \frac{f_{o}}{\overline{f_{1}}} \approx 2 \mathrm{~m} \Omega,
$$

i.e., 2 orders of magnitude smaller than the LHC impedance budget ( $n$ is the harmonic number $n=f / f_{o}$ ). At the resonator frequency $\overline{f_{1}}$, the impedance $|Z / n|$ is real and equal to that of Eq. (32) multiplied by the $Q$ factor.

An estimate of the longitudinal instability threshold (for mode numbers of the order of $\overline{f_{1}} / f_{\text {bunch }}=130$ ) can be done by using the Boussard criterion [13], derived from the coasting beam theory, which we write here in the form

$$
N_{\mathrm{th}}=\frac{(2 \pi)^{(3 / 2)}\left(E_{0} / e\right) \alpha_{c} \sigma \sigma_{\varepsilon 0}^{2}}{c e|Z / n|},
$$

with $E_{0}$ the nominal energy, $e$ the electron charge, $\alpha_{c}$ the momentum compaction, $\sigma_{\varepsilon 0}$ the energy spread, and $Z$ the coupling impedance at a frequency corresponding to harmonic number $n$. For the top energy of $7 \mathrm{TeV}$ we get

$$
N_{\text {th }}=\frac{7.22 \times 10^{11}}{\left|\frac{Z / n}{\Omega}\right|}
$$

TABLE I. LHC parameter list for two operating conditions, namely, at top energy (top) and at injection energy (inj.).

\begin{tabular}{lccr}
\hline \hline Momentum compaction $\left(10^{-4}\right)$ & $\alpha_{c}$ & 3.47 & \\
Machine length $(\mathrm{km})$ & $L_{o}$ & 26.66 & \\
Revolution frequency (kHz) & $f_{o}$ & 11 & \\
Average beta function (m) & $\bar{\beta}$ & 100 & \\
Energy (GeV) & $E_{o} 7 \times 10^{3}$ (top) & 450 (inj.) \\
Bunch length (mm) & $\sigma$ & 75 (top) & 130 (inj.) \\
Energy spread $\left(10^{-4}\right)$ & $\sigma_{\varepsilon o}$ & 1.1 (top) & 4.7 (inj.) \\
Synchrotron tune $\left(10^{-3}\right)$ & $Q_{s}$ & 2.12 (top) & 6 (inj.) \\
\hline \hline
\end{tabular}


protons per bunch, while in the case of injection energy $(450 \mathrm{GeV})$, the threshold is a factor of 2 higher.

In the most pessimistic case in which the perturbation of the unstable oscillation mode has the same resonant frequency $\overline{f_{1}}$ of the wake, we obtain a threshold of

$$
N_{\text {th }}=\frac{3.37 \times 10^{14}}{Q}
$$

protons per bunch, depending on the value of $Q$. Quality factors $Q$ higher than $2 \times 10^{3}$ (equivalent to effective interaction lengths longer than about $4 \mathrm{~m}$ ) are therefore dangerous for the LHC, whose ultimate intensity is $1.6 \times$ $10^{11}$ protons per bunch.

On one hand, a more accurate stability analysis including azimuthal [12] and radial [14] mode coupling would then be in order. On the other hand, several mechanisms, not included in the previous derivation, will limit the quality factor $Q$, including Ohmic losses and geometric imperfections. In particular, pumping slots in the LHC beam screen induce mixing of the modes propagating in the beam pipe and attenuation of the synchronous surface wave, presumably after a distance of a few meters [15].

\section{B. Application to the LCLS undulator}

The longitudinal wake discussed so far is relevant also for very short bunches; an interesting example is the LCLS undulator [8], where the expected bunch is short (the rms bunch length is $\sigma=15 \mu \mathrm{m}$ ) and rather rectangular in shape. For a rectangular charge distribution, with half width $T=\sqrt{3} \sigma / c$, the rms energy spread (over the whole machine) is simply given by [16]

$$
\begin{aligned}
\frac{\Delta E^{\mathrm{rms}}}{E}= & \frac{N q L_{c} w_{0}(a, b, h)}{2 E} \\
& \times \sqrt{\frac{1}{2\left(\omega_{1} T\right)^{2}}\left[1-\frac{\sin \left(4 \omega_{1} T\right)}{4 \omega_{1} T}\right]-\left[\frac{\sin \left(\omega_{1} T\right)}{\omega_{1} T}\right]^{4}},
\end{aligned}
$$

where $E$ is the energy, $L_{c}$ is the length of the machine, $N q$ is the bunch charge, and $\omega_{1}=2 \pi \bar{f}_{1}$. Figure 4 shows the result for a geometry similar to the LCLS undulator (the circular beam pipe of radius $r=2.5 \mathrm{~mm}$ is approximated by a square one with a side of $a=4 \mathrm{~mm}$ ). Thus, concerning the effect of a synchronous mode, the requirement of having a relative energy spread increase smaller than $5 \times 10^{-4}$ is satisfied if the beam pipe roughness is kept below $10 \mu \mathrm{m}$. Applying the dielectric layer model with an analogous choice of parameters, Ref. [16] found a

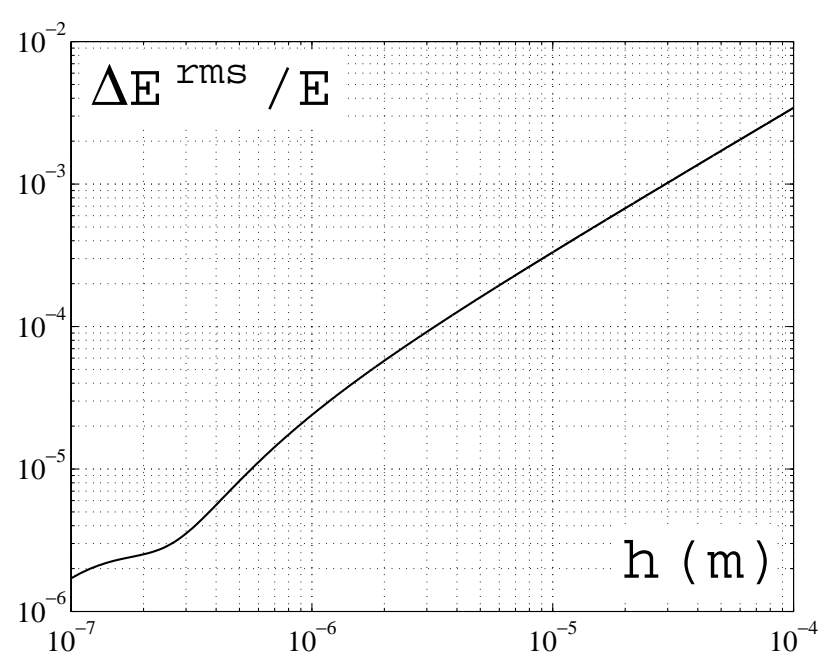

FIG. 4. Energy spread for a geometry approximating the LCLS undulator as a function of the depth of the corrugation $(a=b=$ $4 \mathrm{~mm}, L_{c}=112 \mathrm{~m}, N q=1 \mathrm{nC}, E=14.3 \mathrm{GeV}$, and $\sigma=$ $15 \mu \mathrm{m})$.

maximum allowed corrugation depth of $100 \mathrm{~nm}$, technically not easy to achieve. This result differs by 2 orders of magnitude with ours and such a discrepancy cannot be explained only by the different model of the pipe (we approximate a circular beam pipe with a square one). Our method applied to a circular beam pipe (more similar to the real shape of LCLS ) gives an energy spread even smaller of about a factor of 3-4 than the one discussed here [17].

\section{TRANSVERSE WAKE FUNCTION AND COUPLING IMPEDANCE}

We now consider a point charge moving on a trajectory parallel to the $z$ axis vertically displaced by $y_{0}$. The crossing frequency $\overline{f_{1, \perp}}$ is different from the previous one, as shown in Eq. (E4); the electromagnetic field components are reported as well in Appendix E.

To derive the (specific) longitudinal and transverse dipole impedance, we follow a way slightly different from that of Sec. V, simply applying the definitions reported in [10]. Longitudinal and transverse wake functions are proportional to the Lorentz force; in the $z$ direction

$$
\frac{d w(x, y, z ; \tau)}{d z}=-\frac{F(x, y, z ; \tau)}{q_{1} q},
$$

where $F$ is the Lorentz force in the $z$ direction, $q$ is the trailing charge, and $q_{1}$ the leading one. Approximating for ultrarelativistic particles and for small $h$ and $y_{0}$ at the lowest frequency $(n=1)$, we find

$$
\frac{d w^{\text {dipole }}(x, y, z ; \tau)}{d z}=4 \pi Z_{0} c \frac{h}{a} \frac{y_{0}}{a} \frac{1}{a b}\left\{\tanh \left(\frac{\pi b}{2 a}\right)\left[\frac{\sinh (\pi b / a)}{\pi b / a}\right]\right\}^{-1} \cos \left(\frac{\pi x}{a}\right) \sinh \left(\frac{\pi y}{a}\right) \cos \left(2 \pi \overline{f_{1, \perp}} \tau\right) H(\tau)
$$


after a Fourier transform, we get the dipole component of the longitudinal impedance per unit length:

$$
\begin{aligned}
\frac{d Z^{\text {dipole }}(x, y, z ; \omega)}{d z}= & 4 \pi^{2} Z_{0} \frac{h}{a} \frac{y_{0}}{a} \frac{1}{a b}\left\{\tanh \left(\frac{\pi b}{2 a}\right)\left[\frac{\sinh (\pi b / a)}{\pi b / a}\right]\right\}^{-1} \\
& \times\left[\delta\left(\frac{\omega}{c}-\xi_{z 1, \perp}\right)+\delta\left(\frac{\omega}{c}+\xi_{z 1, \perp}\right)\right] \cos \left(\frac{\pi x}{a}\right) \sinh \left(\frac{\pi y}{a}\right) .
\end{aligned}
$$

Similarly, the transverse wake function per unit length is defined as

$$
\frac{d w_{y}(x, y, z ; \tau)}{d z}=\frac{F_{y}(x, y, z ; \tau)}{q_{1} q}
$$

where $F_{y}$ is the Lorentz force in the $y$ direction; in the usual approximations it becomes

$$
\begin{aligned}
\frac{d w_{y}(x, y, z ; \tau)}{d z}= & -4 \pi^{3 / 2} Z_{0} c\left(\frac{h}{a}\right)^{3 / 2} \frac{y_{0}}{a} \frac{1}{a b}\left\{\left[\tanh \left(\frac{\pi b}{2 a}\right)\right]^{3 / 2}\left[\frac{\sinh (\pi b / a)}{\pi b / a}\right]\right\}^{-1} \\
& \times \cos \left(\frac{\pi x}{a}\right) \cosh \left(\frac{\pi y}{a}\right) \sin \left(2 \pi \overline{f_{1, \perp}} \tau\right) H(\tau) .
\end{aligned}
$$

The transverse impedance $Z_{y}$ is defined as the Fourier transform of the transverse wake function (in the frequency domain) times the imaginary unit [see Eq. (97) in Ref. [10] ]; thus,

$$
\begin{aligned}
\frac{d Z_{y}(x, y, z ; \omega)}{d z}= & -j 4 \pi^{5 / 2} Z_{0}\left(\frac{h}{a}\right)^{3 / 2} \frac{y_{0}}{a} \frac{1}{a b}\left\{\left[\tanh \left(\frac{\pi b}{2 a}\right)\right]^{3 / 2}\left[\frac{\sinh (\pi b / a)}{\pi b / a}\right]\right\}^{-1} \\
& \times\left[\delta\left(\frac{\omega}{c}-\xi_{z 1, \perp}\right)-\delta\left(\frac{\omega}{c}+\xi_{z 1, \perp}\right)\right] \cos \left(\frac{\pi x}{a}\right) \cosh \left(\frac{\pi y}{a}\right) .
\end{aligned}
$$

Usually the dipole component of the transverse kick is the dominant term for ultrarelativistic particles. Since this term is proportional to the displacement $y_{0}$ of the charge $q_{1}$, it is usually defined as a transverse dipole wake function (impedance) as a transverse wake (impedance) per unit of transverse displacement. This can be easily obtained from our formulas simply dividing them by $y_{0}$ resulting in the usual units for transverse dipole wake function $(\mathrm{V} / \mathrm{Cm})$ and impedance $(\Omega / \mathrm{m})$.

\section{Application to the LHC beam screen}

For the evaluation of the effect of the surface roughness wakefields on the transverse dynamics, we use the theory of transverse mode coupling instability, which occurs when the frequencies of two neighboring head tail modes approach each other due to the detuning caused by the current. The instability threshold is given by [18]:

$$
N_{\mathrm{th}}^{\perp}=\frac{2 \pi\left(E_{0} / e\right) Q_{s} F(\sigma)}{\overline{f_{1, \perp}} \bar{\beta} e\left(R_{s}^{\perp} / Q\right)},
$$

where the transverse wake frequency $\overline{f_{1, \perp}}$ is $79 \mathrm{GHz}$, according to Eq. (E4). $F(\sigma)$ is a form factor which, for short bunches, is $\approx 1$; for longer bunches, it increases proportionally to $\sigma$ [19]. We consider the most pessimistic case with $F(\sigma)=1$. For calculating the shunt impedance $R_{s}^{\perp}$, we use the same hypothesis of the longitudinal case, i.e., we assume a high $Q$ transverse resonator with a shunt resistance given by

$$
R_{s}^{\perp}=Q \frac{w_{0 y}^{\perp} L_{0}}{2 \pi \overline{f_{1, \perp}}} \approx 10^{4} Q \Omega / \mathrm{m}
$$

where $w_{0 y}^{\perp}$ is the amplitude of the transverse wake function per unit length of Eq. (41) $\left(w_{0 y}^{\perp}=0.20 \mathrm{~V} \mathrm{pC}^{-1} \mathrm{~m}^{-2}\right)$. By using parameters from Table I in Eq. (43), we get a threshold value of $7.4 \times 10^{12}\left(1.34 \times 10^{12}\right)$ protons per bunch at top (injection) energy, which is much higher than the respective longitudinal one.

\section{CONCLUSIONS}

In this paper we have derived the longitudinal and the transverse wakes due to a periodic corrugation in a rectangular beam pipe. For a point charge, the amplitude of the sinusoidal wake function is proportional to the corrugation depth $h$ and the oscillation frequency is proportional to $1 / \sqrt{h}$. For $h \rightarrow 0$ the frequency of the wake function goes to infinity, while its amplitude vanishes. This last result disagrees from the one obtained in [6] with a dielectric layer model, where the amplitude of the wake does not depend on the corrugation depth. The results reported in [6] are also difficult to apply directly. Computer simulations are needed to establish the correct value of the parameter of the equivalent dielectric layer (i.e., its dielectric constant since its thickness is chosen equal to $h$ ). Those simulations are also not easy for very small corrugation depth and they need, in principle, to be repeated for any change in the sizes of a given geometry. 
Application of our results to the case of the LHC beam screen indicates that the $Q$ factor of the synchronous surface wave should not exceed 2000 to ensure longitudinal beam stability. In the case of the LCLS undulator, a beam pipe roughness below $10 \mu \mathrm{m}$ is acceptable.

\section{ACKNOWLEDGEMENT}

The authors are grateful to Professor F. Frezza and Dr. J. Gareyte for useful discussions.

\section{APPENDIX A}

The magnetic Hertz potential $\Pi_{x}$ satisfies the wave equation, thus

$$
\begin{aligned}
\Pi_{x}= & {\left[A \cos \left(\xi_{x} x\right)+B \sin \left(\xi_{x} x\right)\right] } \\
& \times\left[C \cos \left(\xi_{y} y\right)+D \sin \left(\xi_{y} y\right)\right] e^{-j \xi_{z} z},
\end{aligned}
$$

where $A, B, \xi_{x}$, and $\xi_{y}$ are constants. Since the tangential electric field vanishes on the boundary $x= \pm a / 2$, the four possible expressions are

$$
\begin{array}{rlrl}
\Pi_{x 1} & =\sum_{n} A_{n} \cos \left(\frac{n \pi}{a} x\right) \cos \left(\xi_{y} y\right) e^{-j \xi_{z n} z} & n & =1,3,5, \ldots, \\
\Pi_{x 2} & =\sum_{n} A_{n} \cos \left(\frac{n \pi}{a} x\right) \sin \left(\xi_{y} y\right) e^{-j \xi_{z n} z} & n & =1,3,5, \ldots, \\
\Pi_{x 3} & =\sum_{n} A_{n} \sin \left(\frac{n \pi}{a} x\right) \cos \left(\xi_{y} y\right) e^{-j \xi_{z n} z} & n & =0,2,4, \ldots, \\
\Pi_{x 4} & =\sum_{n} A_{n} \sin \left(\frac{n \pi}{a} x\right) \sin \left(\xi_{y} y\right) e^{-j \xi_{z n} z} & n & =0,2,4, \ldots,
\end{array}
$$

where $A_{n}$ depends on the source and $\xi_{y}$ is related to the other boundary condition. For instance, in a smooth guide, the electric field vanishes also on the boundary $y= \pm b / 2$ leading to $\xi_{y}=k_{y}=m \pi / b$ with $m$ an even (odd) integer number for $\Pi_{x 1}$ and $\Pi_{x 3}\left(\Pi_{x 2}\right.$ and $\left.\Pi_{x 4}\right)$.

\section{APPENDIX B}

In this appendix we derive Eq. (13), showing that $\xi_{z}$ is the sum of the wave number of a smooth guide $\left(k_{z}\right)$ plus a term depending on the corrugation depth $h$ and vanishing for $h \rightarrow 0$.

Considering the roughness as a perturbation of a smooth waveguide, $\xi_{y}$ can be written as

$$
\xi_{y}=k_{y}+\delta k_{y},
$$

where $\delta k_{y}$ is the difference from the unperturbed $k_{y}=m \pi / b$ and it vanishes for $h \rightarrow 0$. We may write Eq. (6) as

$$
\begin{aligned}
K_{y n} \tan \left(K_{y n} h\right) & =\left(k_{y}+\delta k_{y}\right) \frac{\cos (m \pi / 2) \cos \left(\delta k_{y} b / 2\right)-\sin (m \pi / 2) \sin \left(\delta k_{y} b / 2\right)}{\sin (m \pi / 2) \cos \left(\delta k_{y} b / 2\right)+\cos (m \pi / 2) \sin \left(\delta k_{y} b / 2\right)} \\
& =-\left(k_{y}+\delta k_{y}\right) \tan \left(\delta k_{y} b / 2\right),
\end{aligned}
$$

having to consider only odd values for $m$ since Eq. (6) is obtained from $\Pi_{x 2}$. Therefore, using $\tan (x) \approx x$ and solving a second order algebraic equation, we get

$$
\delta k_{y}=-\frac{k_{y}}{2} \pm \sqrt{\left(\frac{k_{y}}{2}\right)^{2}-2 \frac{K_{y n}}{b} \tan \left(K_{y n} h\right)},
$$

were only the + sign is physically meaningful. Same arguments (and same result) hold in simplifying Eq. (E3) for $m$ even. Therefore the wave number becomes

$$
\xi_{z}^{2}=k^{2}-k_{x}^{2}-\xi_{y}^{2} \approx k^{2}-k_{x}^{2}-k_{y}^{2}-2 k_{y} \delta k_{y},
$$

and then

$$
\xi_{z}^{2} \approx k^{2}-k_{x}^{2}-k_{y}^{2} \sqrt{1-8 \frac{K_{y n}}{b k_{y}^{2}} \tan \left(K_{y n} h\right)} \approx k_{z}^{2}+\frac{4 K_{y n}}{b} \tan \left(K_{y n} h\right),
$$

eventually taking the first order term of the square root, we get Eq. (13). 


\section{APPENDIX C}

In this Appendix we derive Eqs. (14). The Lorentz reciprocity theorem [11] states that

$$
\oint_{S}\left(\boldsymbol{E}_{n}^{ \pm} \times \boldsymbol{H}-\boldsymbol{E} \times \boldsymbol{H}_{n}^{ \pm}\right) \cdot \boldsymbol{n} d S=\int_{V} \boldsymbol{J} \cdot \boldsymbol{E}_{n}^{ \pm} d V,
$$

where $\boldsymbol{E}, \boldsymbol{H}$ is the field radiated by the source of current density $\boldsymbol{J}$ in the volume $V$ closed in the surface $S$ with inward normal $\boldsymbol{n} . \boldsymbol{E}_{n}, \boldsymbol{H}_{n}$ are the normal modes of the structure; that is

$$
\begin{aligned}
\boldsymbol{E}_{n}^{ \pm} & =\boldsymbol{e}_{n}^{ \pm} e^{\mp \Gamma_{n} z}=\left(\boldsymbol{e}_{t n} \pm \boldsymbol{e}_{z n}\right) e^{\mp \Gamma_{n} z} \text { and } \\
\boldsymbol{H}_{n}^{ \pm} & =\boldsymbol{h}_{n}^{ \pm} e^{\mp \Gamma_{n} z}=\left( \pm \boldsymbol{h}_{t n}+\boldsymbol{h}_{z n}\right) e^{\mp \Gamma_{n} z} .
\end{aligned}
$$

The index \pm stands for forward and backward propagating waves with respect to a fixed origin $z=0$. The field radiated in the positive $z$ direction by the current filament $\boldsymbol{J}$ can be represented by

$$
\boldsymbol{E}^{+}=\sum_{n} a_{n} \boldsymbol{E}_{n}^{+}, \quad \boldsymbol{H}^{+}=\sum_{n} a_{n} \boldsymbol{H}_{n}^{+},
$$

while the field propagating in the negative $z$ direction is

$$
\boldsymbol{E}^{-}=\sum_{n} b_{n} \boldsymbol{E}_{n}^{-}, \quad \boldsymbol{H}^{-}=\sum_{n} b_{n} \boldsymbol{H}_{n}^{-} .
$$

For any perfectly conducting cylindrical waveguide, the integral over $S$ reduces to one over the generic cross sections $S_{1}$ and $S_{2}$ :

$$
\oint_{S} \ldots \cdot \boldsymbol{n} d S=\int_{S_{1}} \ldots \cdot \boldsymbol{n}_{1} d S_{1}+\int_{S_{2}} \ldots \cdot \boldsymbol{n}_{2} d S_{2},
$$

where $\boldsymbol{n}_{1}=\hat{z}_{o}$ and $\boldsymbol{n}_{2}=-\hat{z}_{o}$. Specifically Eq. (C3) gives the radiated field on the cross section $S_{2}$, while Eq. (C4) gives the field on $S_{2}$. Substituting Eqs. (C3) and (C4), Eq. (C1) becomes

$$
\begin{aligned}
& \int_{S_{1}}\left(\boldsymbol{E}_{n}^{+} \times \sum_{m} b_{m} \boldsymbol{H}_{m}^{-}-\sum_{m} b_{m} \boldsymbol{E}_{m}^{-} \times \boldsymbol{H}_{n}^{+}\right) \cdot \hat{z}_{0} d S_{1}+ \\
& -\int_{S_{2}}\left(\boldsymbol{E}_{n}^{+} \times \sum_{m} a_{m} \boldsymbol{H}_{m}^{+}-\sum_{m} a_{m} \boldsymbol{E}_{m}^{+} \times \boldsymbol{H}_{n}^{+}\right) \cdot \hat{z}_{0} d S_{2}=\int_{V} \boldsymbol{J} \cdot \boldsymbol{E}_{n}^{+} d V,
\end{aligned}
$$

thus, since

$$
\begin{aligned}
& \hat{z}_{0} \cdot \boldsymbol{e}_{i}^{ \pm} \times \boldsymbol{h}_{j}^{\mp}=\mp \hat{z}_{0} \cdot \boldsymbol{e}_{t i} \times \boldsymbol{h}_{t j}, \\
& \hat{z}_{0} \cdot \boldsymbol{e}_{i}^{ \pm} \times \boldsymbol{h}_{j}^{ \pm}= \pm \hat{z}_{0} \cdot \boldsymbol{e}_{t i} \times \boldsymbol{h}_{t j},
\end{aligned}
$$

we get eventually

$$
\begin{aligned}
& -\sum_{m} b_{m} e^{-\left(\Gamma_{n}-\Gamma_{m}\right) z} \int_{S_{1}} \hat{z}_{0} \cdot\left(\boldsymbol{e}_{t n} \times \boldsymbol{h}_{t m}+\boldsymbol{e}_{t m} \times \boldsymbol{h}_{t n}\right) d S_{1}+ \\
& \quad-\sum_{m} a_{m} e^{-\left(\Gamma_{n}+\Gamma_{m}\right) z} \int_{S_{2}} \hat{z}_{0} \cdot\left(\boldsymbol{e}_{t n} \times \boldsymbol{h}_{t m}-\boldsymbol{e}_{t m} \times \boldsymbol{h}_{t n}\right) d S_{2}=\int_{V} \boldsymbol{J} \cdot \boldsymbol{E}_{n}^{+} d V,
\end{aligned}
$$

that is, Eq. (14a) with Eq. (16) and $\Gamma=j \xi_{z}$. With the same reasoning, Eq. (14b) can be derived from Eq. (C1). The integrals defining $A_{n m}$ and $B_{n m}$ should be performed on the whole section of the rough waveguide; nevertheless in our working hypothesis, the transversal field inside the slots is null, thus giving no contribution.

For a smooth waveguide, where the modes are orthogonal, it holds

$$
\int_{S} \boldsymbol{e}_{t i} \times \boldsymbol{h}_{t j} \cdot \hat{z}_{0} d S=\delta_{i j}
$$

leading to Eq. (17).

\section{APPENDIX D}

In this appendix we derive Eq. (21) for the electric field from Eq. (18). Being

$$
\begin{aligned}
A_{n n} & =2 \int_{S} \hat{z}_{0} \cdot \boldsymbol{e}_{t n} \times \boldsymbol{h}_{t n} d S \\
& =2 \int_{S} \hat{z}_{0} \cdot\left[\left(\boldsymbol{e}_{x n}+\boldsymbol{e}_{y n}\right) \times\left(\boldsymbol{h}_{x n}+\boldsymbol{h}_{y n}\right)\right] d S \\
& =-2 \int_{S} e_{y n} h_{x n} d S,
\end{aligned}
$$

from Eq. (3) with $\Pi_{x 2}$, it holds

$$
\begin{gathered}
e_{y n}=-\omega \mu_{0}\left|\xi_{z n}\right| \cos \left(k_{x n} x\right) \sin \left(\xi_{y} y\right), \\
h_{x n}=\left(k^{2}-k_{x n}^{2}\right) \cos \left(k_{x n} x\right) \sin \left(\xi_{y} y\right),
\end{gathered}
$$

and Eq. (19) comes directly. Also Eq. (20) is straightforward since

$$
e_{z}=j \omega \mu_{0} \xi_{y} \cos \left(k_{x n} x\right) \cos \left(\xi_{y} y\right),
$$


and

$$
\int_{-\infty}^{+\infty} e^{-j\left(\omega / \beta c \pm \xi_{z n}\right) z} d z=2 \pi \delta\left(\omega / \beta c \pm \xi_{z n}\right) .
$$

The $z$ component of the electric field $E_{z}$, according to Eqs. (C3) and (C4) is

$$
E_{z}=a_{n} e_{z n} e^{-j \xi_{z n} z}-b_{n} e_{z n} e^{j \xi_{z n} z}
$$

that is, after simple algebraic manipulation Eq. (21).

\section{APPENDIX E}

In this appendix we apply our theory to solve the transverse problem. We consider a point charge moving on a trajectory parallel to the $z$ axis, vertically displaced by $y_{0}$. Analogously to the longitudinal case, we work with the magnetic Hertz potential; due to the dipole symmetry, we choose the first magnetic Hertz potential (see Appendix A):

$$
\begin{aligned}
\Pi_{x 1} & =\sum_{n} A_{n} \cos \left(\frac{n \pi}{a} x\right) \cos \left(\xi_{y} y\right) e^{-j \xi_{z n} z} \\
n & =1,3,5, \ldots
\end{aligned}
$$

The fields, as calculated from Eq. (3), are

$$
e_{x}=0
$$

$$
\begin{aligned}
e_{y} & =-\omega \mu_{0} \xi_{z} \cos \left(\frac{n \pi}{a} x\right) \cos \left(\xi_{y} y\right), \\
e_{z} & =-j \omega \mu_{0} \xi_{y} \cos \left(\frac{n \pi}{a} x\right) \sin \left(\xi_{y} y\right), \\
h_{x} & =\left[k^{2}-\left(\frac{n \pi}{a}\right)^{2}\right] \cos \left(\frac{n \pi}{a} x\right) \cos \left(\xi_{y} y\right), \\
h_{y} & =\left(\frac{n \pi}{a}\right) \xi_{y} \sin \left(\frac{n \pi}{a} x\right) \sin \left(\xi_{y} y\right), \\
h_{z} & =j\left(\frac{n \pi}{a}\right) \xi_{z} \sin \left(\frac{n \pi}{a} x\right) \cos \left(\xi_{y} y\right) .
\end{aligned}
$$

The boundary conditions at the walls lead to the following dispersion equation:

$$
K_{y n} \tan \left(K_{y n} h\right)=-\xi_{y} \tan \left(\xi_{y} \frac{b}{2}\right),
$$

the cutoff frequencies of the modes are given by Eq. (12) with even values of $m$ (the lowest dipole mode is $\mathrm{TE}_{10}$ whose cutoff does not depend on $h$ ). Following the same steps used to derive Eq. (10), the crossing frequencies (where $\xi_{z}=\omega / c$ ) are

$$
\overline{f_{n, \perp}}=\frac{c}{2 \pi} \sqrt{k_{x n}^{2}+\frac{k_{x n}}{h} \tanh \left(k_{x n} \frac{b}{2}\right)}
$$

giving $79 \mathrm{GHz}$ for the first $(n=1)$ crossing frequency in a LHC-like geometry $\left(a=3.6 \times 10^{-2} \mathrm{~m}, b=4.3 \times\right.$ $10^{-2} \mathrm{~m}$, and $\left.h=30 \mu \mathrm{m}\right)$ and for an off-axis charge. Applying the reciprocity relations [see Eq. (14)], we get

$$
\begin{aligned}
& E_{z}(x, y, z ; \omega)=-2 \pi \beta \frac{q Z_{0}\left[\delta\left(\frac{\omega}{\beta c}-\xi_{z n}\right)+\delta\left(\frac{\omega}{\beta c}+\xi_{z n}\right)\right]}{a\left[b / 2+\sin \left(\xi_{y} b\right) / 2 \xi_{y}\right]} \frac{\xi_{y}^{2}}{k^{2}-k_{x n}^{2}} \sin \left(\xi_{y} y_{0}\right) \cos \left(k_{x n} x\right) \sin \left(\xi_{y} y\right) e^{-j z \omega / \beta c}, \\
& E_{y}(x, y, z ; \omega)=-j 2 \pi \beta \frac{q Z_{0}\left[\delta\left(\frac{\omega}{\beta c}-\xi_{z n}\right)-\delta\left(\frac{\omega}{\beta c}+\xi_{z n}\right)\right]}{a\left[b / 2+\sin \left(\xi_{y} b\right) / 2 \xi_{y}\right]} \frac{\xi_{y} \omega / c}{k^{2}-k_{x n}^{2}} \sin \left(\xi_{y} y_{0}\right) \cos \left(k_{x n} x\right) \cos \left(\xi_{y} y\right) e^{-j z \omega / \beta c},
\end{aligned}
$$

$$
H_{x}(x, y, z ; \omega)=j 2 \pi \beta \frac{q\left[\delta\left(\frac{\omega}{\beta c}-\xi_{z n}\right)-\delta\left(\frac{\omega}{\beta c}+\xi_{z n}\right)\right]}{a\left[b / 2+\sin \left(\xi_{y} b\right) / 2 \xi_{y}\right]} \frac{\xi_{y} c}{\omega} \sin \left(\xi_{y} y_{0}\right) \cos \left(k_{x n} x\right) \cos \left(\xi_{y} y\right) e^{-j z \omega / \beta c}
$$

These expressions are used in Sec. VI to derive the dipole wake function and coupling impedance.

[1] O. Brüning et al., in Proceedings of the IEEE Particle Accelerator Conference, New York, 1999, edited by A. Luccio and W. MacKay (IEEE, Piscataway, NJ, 1999), pp. 2629-2631.
[2] S. Kurennoy, Phys. Rev. E 55, 3529 (1997).

[3] G. Stupakov, Phys. Rev. ST Accel. Beams 1, 064401 (1998).

[4] M. Chatard-Moulin and A. Papiernik, IEEE Trans. Nucl. Sci. 26, 3523 (1979).

[5] R. L. Gluckstern and S. Krinsky, IEEE Trans. Nucl. Sci. 28, 2621 (1981).

[6] M. Timm, A. Novokhatski, and T. Weinland, in 
Proceedings of the International Computational Accelerator Physics Conference, Monterey, California, 1998 (Stanford Linear Accelerator Center, Menlo Park, CA, 1998).

[7] G. Di Massa, CERN Report No. SPS/ARF Note 85-8, 1985 (unpublished).

[8] The LCLS Design Study Group, SLAC Report No. SLAC-R-521 (unpublished).

[9] G. H. Bryant, Proc. IEE 116, 203 (1969).

[10] L. Palumbo, V. G. Vaccaro, and M. Zobov, in Proceedings of the CERN Accelerator School: Advanced Accelerator Physics Course, Rhodes, 1993, edited by S. Turner (CERN Yellow Report No. 95-06) (European Laboratory for Particle Physics, Geneva, Switzerland, 1995), pp. 331-390.

[11] R. Collin, Field Theory of Guided Waves (Oxford University Press, Oxford, 1995), 2nd ed.

[12] A.W. Chao, Physics of Collective Beam Instabilities in High Energy Accelerators (Wiley-Interscience, New York, 1993).
[13] D. Boussard, CERN Report No. LABII/RF/Int./75-2, 1975 (unpublished).

[14] A. W. Chao, B. Chen, and K. Oide, Report No. SLACPUB-6859, 1995; Proceedings of the IEEE Particle Accelerator Conference, Dallas, 1995 (IEEE, Piscataway, NJ, 1996), pp. 3040-3042.

[15] F. Caspers (private communication).

[16] K.L.F. Bane and A. Novokhatski, SLAC Report No. SLAC-AP-117 [and also Report No. LCLS-TN-99-1, 1999 (unpublished)].

[17] M. Angelici, F. Frezza, A. Mostacci, and L. Palumbo, in Proceedings of the 7th European Particle Accelerator Conference, Vienna, Austria, 2000, http://accelconf.web. cern.ch/AccelConf/e00/index.html, pp. 1438-1440.

[18] F. Ruggiero, CERN Report No. 86-06, 1986 (unpublished).

[19] A. W. Chao and M. Tigner, Handbook of Accelerator Physics and Engineering (World Scientific, Singapore, 1999). 